\title{
Governing Sustainable Development Goals: interactions, infrastructures, and institutions
}

\author{
Jeff Waage ${ }^{*}$ Christopher Yap ${ }^{\dagger}$, Sarah Bell ${ }^{*}$, \\ Caren Levy ${ }^{\S}$, Georgina Mace ${ }^{\S}$ Tom Pegram ${ }^{* *}$, \\ Elaine Unterhalter ${ }^{\dagger \dagger}$, Niheer Dasandi ${ }^{* *}$, \\ David Hudson ${ }^{* *}$, Richard Kock ${ }^{\ddagger}$, \\ Susannah H. Mayhew ${ }^{\S \S}$, Colin Marx \\ and Nigel Poolesg
}

${ }^{\star}$ London International Development Centre; School of Oriental and African Studies, Centre for Development, Environment and Policy, ${ }^{\dagger}$ London International Development Centre; University College London, Bartlett Development Planning Unit, *University College London, Centre for Environmental and Geomatic Engineering, 'University College London, Bartlett Development Planning Unit, "University College London, Centre for Biodiversity and Environment Research, ${ }^{*}$ University College London, School of Public Policy, ${ }^{\dagger \dagger}$ University College London, Institute of Education, Department

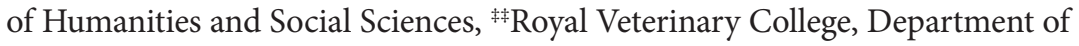
Pathology and Pathogen Biology, ${ }^{s 5}$ London School of Hygiene and Tropical Medicine, Department of Global Health and Development, "School of Oriental and African Studies, Centre for Environment, Development and Policy

\section{Introduction}

Sustainable development is hard to define and harder to achieve. The process of expansion from eight MDGs with 18 targets and 48 indicators, to proposals

How to cite this book chapter:

Waage, J, Yap, C, Bell, S, Levy, C, Mace, G, Pegram, T, Unterhalter, E, Dasandi, N, Hudson, D, Kock, R, Mayhew, S. H, Marx, C, and Poole, N. 2015. Governing Sustainable Development Goals: interactions, infrastructures, and institutions. In: Waage, J and Yap, C. (eds.) Thinking Beyond Sectors for Sustainable Development. Pp. 79-88. London: Ubiquity Press. DOI: http://dx.doi.org/10.5334/bao.i 
for 17 SDGs with over 100 indicators, demonstrates the evolving awareness by the international community of the complex nature of development and its implications for society, the economy, and the environment. Whilst the development of goals, targets, and indicators shows a stronger commitment to defining and monitoring constituent elements, sustainable development is more than the sum of its parts. It is an outcome of positive synergies between multiple elements, and may be undermined by negative trade-offs between them.

The proposed SDGs have been informed, influenced, and developed by different sectoral constituencies. As with the MDGs (Waage et al. 2010), this process has not sufficiently addressed the interactions between goals, and between the mechanisms and processes that could be established to achieve them. These interactions could be positive or negative, and the nature of them may be physical, physiological, socio-political, or any combination thereof. The challenge for achieving sustainable development is how to manage and govern these interactions.

The experience of the MDGs shows that strong institutional ownership of goals made them more likely to be delivered. However, while the MDGs were coherent on systems of measurement, they were weak in facilitating participation and voice in relation to reviewing implementation (Hulme 2013). Similar governance mechanisms for the newly proposed SDGs, such as those pertaining to the management of climate change and the environment, have so far proved difficult to establish.

Governance in terms of responsibility, transparency, accountability, capacity, and legitimacy at sub-national, national, and international levels is essential for achieving sustainable development. A successful governance process for the SDGs, which engages with the full range of political interests, would link the delivery of goals that are capable of synergy, and negotiate trade-offs to optimise delivery of goals that are in conflict; governance within silos is no longer tenable.

This chapter proposes a framework for classifying and clustering goals and their interactions, uses this to identify the different problem structures and challenges for governance, and proposes potential solutions. We use this novel conceptualisation to show why different goals interact positively or negatively, and where and why governing these interactions can lead to a 'win-win', as well as where governing these interactions is a much more politically difficult challenge.

\section{Levels of sustainable development}

The framework is based on the SDGs as proposed in the Report of the Open Working Group of the General Assembly on Sustainable Development Goals (2014). It consists of three levels, to which we assign goals based on their intended outcomes. This is illustrated in Figure 2. 


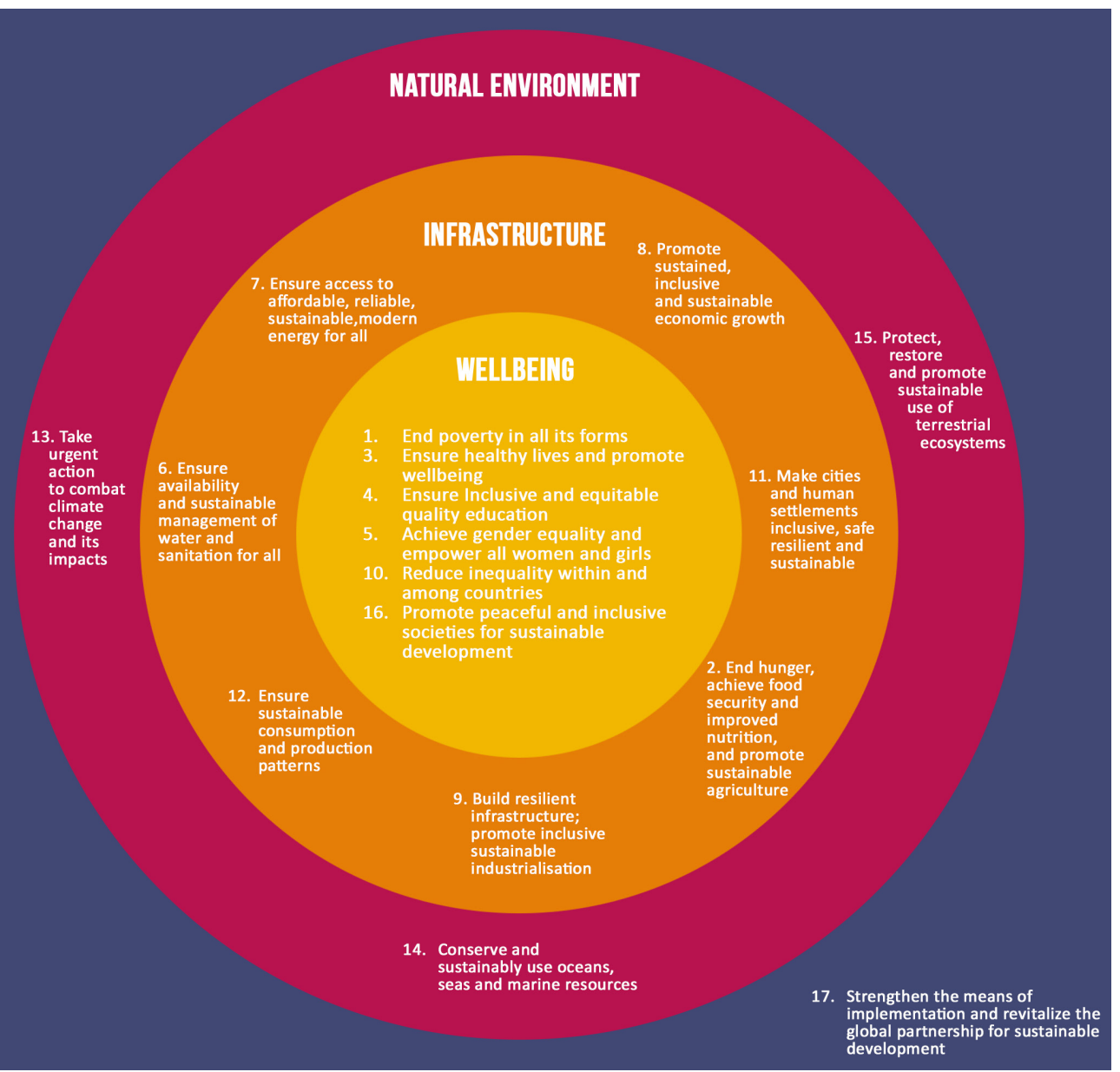

Figure 2: The SDGs as proposed in the Report of the Open Working Group of the General Assembly on Sustainable Development Goals, placed in the proposed framework. ${ }^{8}$

${ }^{8}$ The goals have been paraphrased in the above diagram, for the full list of proposed goals see the Appendix. The placement of goals in particular parts of this diagram is approximate. For a few goals, the proposed sub-goals and targets actually spread across different levels. For instance, the water-related targets of Goal 6 address environmental (water resource), infrastructural (useable capacity), and well-being (sanitation) levels, while the agriculture-related targets of Goal 2 address infrastructural (production) and well-being (hunger) levels. Please note that the concentric levels do not represent geographical scale in a narrow sense, but different ways in which the goals impact. All goals must have mechanisms for delivery on local, national, and global scales. 
The inner level, Well-Being, includes 'people-centred' goals that aim to deliver individual and collective outcomes, such as health, education, and nutrition, which directly pertain to welfare and well-being and their equitable distribution within and between individuals and countries. They represent well-established terrain for governments and are a key component of accountability (the social contract between the state and society) and state legitimation strategies. The MDGs established some minimal lines of delivery for goals of this kind, but the draft SDGs represent a more ambitious attempt to support well-being and welfare in additional areas, such as promoting peaceful and inclusive societies, where governments have less experience.

The middle level, Infrastructure, includes goals that relate to various kinds of networks and mechanisms for the production, distribution, and delivery of goods and services, including food, energy, clean water, and waste and sanitation services in cities and human settlements. These goals transcend individuals, households, and communities, and address many of the perceived essential functions of modern society within and sometimes beyond nation states. They are assumed to contribute to growth in well-being while at the same time reducing intensity of resource use, pollution, and negative impacts on the environment.

The outer level, Environment, groups together those goals that relate primarily to the management of global resources, underlying support systems and global public goods such as land, ocean, air, natural resources, biodiversity, and the management of climate change. Here are the biophysical systems that underpin sustainable development. While not dependent on human activities, these systems are strongly influenced by them. These goals typically require international and transnational cooperation for their realisation.

We have left Goal 17, relating to revitalising the global partnership for sustainable development, outside our three levels because it is a cross-cutting goal relating to goals in all levels.

\section{Interactions}

This framework clarifies the major interactions between groups of goals. Optimally, all goals contribute to improving agreed measures of individual and community well-being (health, education, income, security, and so forth). However, the potential positive and negative interactions between all SDGs are closely associated with their positioning at different levels in our framework.

In general, interactions between goals in the inner level pose opportunities for positive synergies and win-wins, because of their similar relation to notions of human development, and focus on individual and community well-being. Examples include the synergies that could be achieved by aligning the design of health, gender, and education goals to improve sexual and reproductive health (SRH), as well as the empowerment of women and girls, or between nutrition and education goals to improve early years development. The particular example of SRH is developed further in the next chapter. 
Interactions between the inner and middle levels are mutually supportive. The importance of achieving the goals in the middle level to the inner level is clear: improving access to water, food, energy, and so forth, are all necessary to achieve the inner level, people-centred outcomes. However, there is also significant reciprocal influence, such as the positive impact of an educated, healthy labour force on agricultural production. The interactions between the outer and middle levels characterise the ways in which human societies appropriate environmental resources and services. 'Sustainability', a component of several goals at the middle level, is linked to how these environmental services are used.

The complicated and poorly understood links between goals at the outermost and innermost levels mean that the objectives of infrastructural goals at the middle level are often unclear, easily contested, and subject to multiple demands which may be in conflict. For instance, achieving Goal 2 on sustainable agriculture and food security, which will contribute to improving well-being by reducing hunger, may require increased agricultural production with consequential adverse impacts on goals to tackle climate change (Goal 13) and protect marine (Goal 14) and terrestrial (Goal 15) ecosystems. Achieving Goal 7, relating to energy, or Goal 6, relating to wastewater treatment, with their benefits to education, health, and other well-being outcomes, may depend on increased energy generation that may also impact negatively on climate change and water resource management.

As a result, goals in the middle level must mediate potentially negative tradeoffs between outer- and inner-level goals, and may interact negatively with each other as they compete for limited environmental resources, e.g. agriculture versus energy. The mechanisms that will need to be established to achieve the goals in the middle level must relate to goals in both the inner and outer levels, and arbitrate these interactions.

Outer-level goals relating to reducing climate change and safeguarding marine and terrestrial ecosystems, natural resources, and water supplies, are closely interrelated and there are potential positive synergies. For instance, improving forest conservation may reduce climate change, while tackling climate change may reduce the loss of coral reefs which are critical marine resources.

The challenges addressed by the outer-level goals relate largely to the challenges of sustainability imposed by goals in the middle level. More subtle and less direct, but still profound, is the influence of the inner level on the outerlevel goals, such as the impact of increasing access to high-quality education and family planning services on reducing population growth, which otherwise increases demand for natural resources and the strain on the environment. The impact of population growth on the outer level is mitigated through mechanisms at the middle level.

The complexity of the interactions between goals located at different levels highlights the challenge of the SDGs as a global policy framework. At the global scale, there exists no structure, institution, or mechanism for governing these types of interactions, particularly where they involve conflicts. This creates substantial and important governance challenges for the SDGs. 


\section{Governance}

Governance refers to the institutions, mechanisms, or processes backed by political power and/or authority that allow an activity or set of activities to be controlled, influenced, or directed in the collective interest (Baker et al. 2005). From this perspective, governance includes: the responsibility an actor has for controlling, influencing, or directing activities in the collective interest; being held accountable to the collective; and having the capacity, in terms of political power and resources, to direct activities towards the collective interest. Governance refers to, amongst others, hard and soft laws, regulations, agreements, institutions (national, local, and regional government; international bodies; secretariats; civil society; and the private sector), shared norms of behaviour, and the balance of power therein.

It has been proposed that the SDGs might have a single, overarching governance goal (United Nations High-Level Panel of Eminent Persons on the Post-2015 Development Agenda 2012), or alternatively, that each goal should have its own governance structure (Foresti 2014). However, our framework shares more parallels with the notion of polycentric governance, whereby multiple centres of authority with circumscribed but autonomous prerogatives nevertheless operate under an overarching rule system (Ostrom 2010).

The diverse nature of the SDGs requires that governance cannot only be treated as a goal, whether stand-alone or embedded within individual goals, but as transcending existing mechanisms. Governance will need to be a careful political process built around broad-based consensus and collective legitimacy, in order to optimise the delivery of goals. Effective governance systems that deliver all of the SDGs should address the complex interactions outlined above, achieving synergy where goals may interact positively, and resolving conflicts where they may interact negatively.

The framework proposed above helps clarify how and where the SDGs face particular governance challenges. Differences and similarities of governance challenges and opportunities correspond to their placement in our conceptual framework. Overall, the challenge is about adopting and adapting existing institutional structures and socio-political conditions, as well as engaging entirely new mechanisms, stakeholders, and perspectives.

Inner-level goals focused on individual and collective outcomes have similar governance and institutional structures, stemming predominantly from the historical role of the state in relation to the provision of health, education, and welfare, the initiatives of the 1990s, and the experience with the MDGs. Whilst the formal institutional structures may be in place, many developing countries will require continued support to strengthen structures and institutions for inner-level goals in order to govern effectively.

At this inner level, the alignment of comparable goals and their implementation across many of these sectors points to broadly synergistic governance 
opportunities. However, such alignment is often far from politically feasible; it is strongly dependent upon transforming existing social norms and conditions, such as gender inequalities in the public and private spheres, which are entrenched by political and economic relations and will require change over generations.

Moreover, at the inner level, there exists the challenge of connecting the public and the private, which relates to many features in, for example, the gender goal (Goal 5), the education goal (Goal 4) on appreciating cultural diversity, and the poverty goal (Goal 1) on building the resilience of the poor. The issue here is how the state and the market negotiate with the realm of the family, the emotional, and the cultural.

For example, providing information for poor, young women about reproductive rights and enhancing access to contraception requires engaging with young girls, boys, families, communities, faith-based organisations, and schools to better understand adolescent sexuality, gender identities, and relationships, and building supportive connections with the health sector. This entails planning, open discussion of difficult issues, and leadership.

In this realm, the non-governmental or 'third sector' can play a pivotal role. In the fields of SRH and gender, NGOs frequently deliver programmes on issues or to groups that the state sector finds problematic (e.g. abortion and adolescent sexual health) and in which the private sector has little commercial interest (e.g. services for poor or excluded minority groups). In the next chapter, this example of inter-goal governance is used to explore a range of possible governance mechanisms. Middle-level, infrastructure-related goals pose particular governance problems relating to conflicts and trade-offs, often between private and public interests, with multiple stakeholders. The middle level represents a relatively new domain for integrated global development goal setting, and brings with it a number of challenges.

This level is where much of the global economy is concentrated, and typically decisions are taken by a small number of powerful actors across both the public and private sectors, by elites, and by technical experts on behalf of the wider public. ${ }^{9}$ The combination of private interests and weaker accountability mechanisms mean decisions are typically made without consideration of the potential interactions with the inner or outer levels. There is an endemic lack of transparency and accountability to the public and, in the SDGs process, little attention is given to local government and organised local communities.

In addition, over the past few decades there has been a positive normativity around building infrastructure associated with energy, water provision, urban development, and growth as ends in themselves. In many countries this is

\footnotetext{
${ }^{9}$ Whilst most people have first-hand experience of service delivery at the inner-level, associated for example with attending school or receiving healthcare, few have experienced or have knowledge of the decision-making processes, implementation, or delivery of services at the middle level. The goals at this level are typically removed from individual experiences.
} 
associated with widening inequalities, problems of environmental sustainability, and intergenerational inequity. Our framework reiterates the importance of interrogating how infrastructure interacts with other levels, and how this can be governed.

The outer level comprises goals relating to land, sea, air, and biodiversity. The governance and management of each of these raises unique challenges. This level currently has the most fragmented governance and institutional landscape, often involving non-binding international agreements and conventions (e.g. the Convention on Biological Diversity (CBD) and the United Nations Framework Convention on Climate Change (UNFCCC)). Outer-level goal governance is currently weak, and its structures consist largely of monitoring and convening processes only, while incentives for stronger governance at this level are poor. Furthermore, current governance structures are focused on environmental conservation, and do not clearly connect to the objectives of well-being in the inner-level goals.

Goals relating to global public goods and shared common resources represent significant challenges, as they rely on greater levels of cooperation and investment in sectors in which the outputs/rewards are less obviously apparent to the electorate in any single country and/or are over longer time horizons; often a generation or more, and certainly beyond an electoral cycle.

Drawing together the above observations, we conclude that the nature of governance challenges changes as you move outwards from the centre of the framework and its well-being goals. For goals in the inner level, many government instruments already exist for delivery, even if they do not always work efficiently or equitably. As we move outward, these mechanisms disintegrate, conflicts arise, and soft laws prevail. As with interactions between goals, potential governance systems are more likely to be similar between goals operating within the same level. The challenge of governing within levels is about building new relationships and new mechanisms that overcome sectoral and ministerial silos.

On the other hand, the interactions occurring between goals that fall into different levels are markedly more complex, and correspondingly, raise more complex governance challenges. This is a key point revealed by our framework, and remains to be addressed if the goals are to be effectively achieved. The challenge of governing across levels is likely to require innovative forms of collective consensus-building, with the inclusion and participation of new stakeholders, across scales, and across sectors.

\section{The significance of the infrastructure level}

In the context of rapid urbanisation, population growth, climate change, and diminishing resources, the middle, infrastructural level goals represent simultaneously the greatest challenge and the greatest opportunity to achieve the sustainable development agenda. In this, the most neglected level in terms of 
the academic and policy discourse, we find the greatest potential synergies, not only between goals within the level, but also the greatest leverage to positively influence the achievement of goals across levels.

The goals of the outer, environmental level can be achieved only if the middle level is governed effectively. The current approach of attempting to govern the outer level without addressing the middle level has meant that the burden placed on global governance initiatives at the outer level is too great. Outer-level goals also function as the ultimate arbitration on the success or failure of the SDGs in terms of sustainability. Inner-level goals will more likely be achieved at the expense of the outer-level goals until resources are virtually exhausted, ecological tipping points are reached, and ecosystem resilience breached. Whilst monitoring and recording of global scale ecosystems will be necessary to ensure that there really is global sustainability, our analysis suggests that the intractable problems of managing the global environment for the benefit of people can be neatly sidestepped by focusing sustainability targets within middle-level goals.

There is a risk that efforts to achieve middle-level goals will be prone to domination by strong special interest groups and short-termism, and will revert to current centralised public and private governance structures and 'business as usual' approaches. Historically, this is the domain of the technical 'experts', where decisions are made on people's behalf. For these reasons, good governance of goals in the middle level requires the strengthening of local government and for decisions to be brought into the realm of public, democratic debate. The significance of the middle level in terms of its interactions with the inner and outer levels means that decisions ought not to be taken by an unaccountable few. Broad-based consensus based on legitimate political procedures of all concerned parties will be vital for the viability of the SDGs agenda.

\section{Recommendations}

Our analysis leads to a number of specific recommendations for governments in the development of governance structures for the proposed SDGs. Firstly, we urge governments to: devise formal governance mechanisms at the national and sub-national levels that are characterised by deliberation, participation, and transparency in decision-making; engage community organisations who are already mobilised around these issues; and invite democratic debate around middle-level goals and particularly conflicts. Responsibilities should be defined, accountability systems put in place, and human capacities built accordingly.

Secondly, we suggest that it is important to learn from and build upon existing governance mechanisms and institutional arrangements. There are several models in existence that are at least partially successful in international standard setting, which might provide useful mechanisms for such governance, such as the UN-REDD for forest resources management and the International Organisation for Standardisation (ISO) for standards of health and environmental protection. We illustrate some of these in the next chapter. 
Finally, the particular focus of governance and international support by donors should be towards developing the capacity of institutions to operate at the middle, infrastructural level of goals, and to manage, regulate, and govern decision-making and development there.

More generally, we suggest that the diverse nature of the SDGs requires that governance cannot be treated as a goal itself (United Nations High-Level Panel of Eminent Persons on the Post-2015 Development Agenda 2012) or embedded within individual goals (Foresti 2014), but needs to be a careful political process by which the collective delivery of goals at different levels is optimised, providing the necessary broad-based consensus and collective legitimacy required to optimise their delivery. Effective governance systems that deliver all of the SDGs should address the complex interactions outlined above, achieving synergy where goals may interact positively, and resolving conflicts where they may interact negatively.

\section{References}

Baker, A., Hudson, D., and Woodward, R. (2005). 'Introduction', in Baker, A., Hudson, D., and Woodward, R. (eds) Governing Financial Globalisation: International Political Economy and Multi-Level Governance, London: Routledge.

Foresti, M. (2014, February 5). 3 reasons 'governance' should not only be a stand-alone development goal. The Interpreter. Retrieved from http://www. lowyinterpreter.org/post/2014/02/05/3-reasons-governance-should-notbe-a-stand-alone-development-goal.aspx?COLLCC $=602054812 \&$

Hulme, D. (2013). The Post-2015 development agenda: learning from the MDGs. Southern Voice Occasional Paper 2. Dhaka, Bangladesh: Centre for Policy Dialogue.

Open Working Group of the General Assembly on Sustainable Development Goals. (2014). Open Working Group proposal for Sustainable Development Goals document A/68/970. New York: United Nations. Retrieved from http://undocs.org/A/68/970

Ostrom, B. (2010). Beyond Markets and States: Polycentric Governance of Complex Economic Systems. American Economic Review, 100 (June 2010), $1-33$.

United Nations High-Level Panel. (2012). Realising the future we want for all: report to the Secretary General. New York: United Nations.

Waage, J., Banerji, R, Campbell, O, Chirwa, E, Collender, G, Dieltiens, V, Dorward, A, et al (2010). 'The Millennium Development Goals: a crosssectoral analysis and principles for goal setting after 2015' The Lancet, vol 376, no. 9745, 991-1023. DOI: http://dx.doi.org/10.1016/S01406736(10)61196-8 